\title{
Transparencia editorial en revistas científicas mexicanas de educación: hacia una gestión integral de las políticas editoriales en las publicaciones periódicas científicas
}

\author{
María Teresa Fernández Bajón* \\ Jenny Teresita Guerra González**
}

Artículo recibido:

27 de agosto de 2020

Artículo aceptado:

24 de septiembre de 2020

Artículo de investigación

\section{Resumen}

El objetivo principal de esta contribución se enmarca en el estudio sobre la transparencia editorial en las 18 revistas mexicanas de educación indizadas en el Sistema de Clasificación de Revistas Mexicanas de Ciencia y Tecnología del Conacyt. El análisis se sustenta en la comprobación del cumplimiento total, parcial o nulo de 15 criterios definidos por las autoras a partir de la revisión de los Principles of Transparency and Best Practice in Scholarly Publishing, publicado en 2018 por el Comitte on Publication Ethics (COPE), y de las Directrices para autores y traductores de artículos cientificos publicados en inglés, propuesto en el mismo año por la European Association of Science Editors (EASE). De los resultados obtenidos destacamos los siguientes:

* Universidad Complutense de Madrid, España

maitefb@ucm.es

** Instituto de Investigaciones Bibliotecológicas y de la Información, UNAM, México

jenny@iibi.unam.mx

INVESTIGACIÓN BIBLIOTECOLÓGICA, vol. 35, núm. 87, abril/junio, 2021, México, ISSN: 2448-8321 pp. 13-32 
sólo una de las 18 revistas analizadas cumple con los 15 criterios establecidos; cinco publicaciones presentan una alta transparencia editorial al cumplir con 14 criterios, y dos revistas cumplieron únicamente nueve de los criterios de transparencia editorial. Se concluye advirtiendo sobre la necesidad de que la transparencia editorial sea un estándar de cumplimiento obligatorio para la indización o permanencia en sistemas de publicaciones como el de Conacyt.

Palabras clave: Transparencia Editorial; Ética de la Comunicación Científica; Revistas de Educación; Publicaciones Científicas; México

Editorial transparency in Mexican scientific journals on Education: Towards a comprehensive management of editorial policies in scientific periodicals Maria Teresa Fernández Bajón and Jenny Teresita Guerra González

\section{Abstract}

The main objective of this article is to study the publishing transparency within the 18 Mexican Education journals indexed in the CONACYT's Classification System of Mexican Journals of Science and Technology. The analysis is based on the verification of total, partial or null compliance with 15 criteria defined by the authors based on the review of Principles of Transparency and Best Practice in Scholarly Publishing (2018), published by the Committee on Publication Ethics (COPE) and of Guidelines for authors and translators of scientific articles published in English (2018), proposed by the European Association of Science Editors (EASE). Among the results obtained are the following: only one of the 18 journals analyzed meets the 15 established criteria; 5 publications present high publishing transparency by meeting 14 criteria, and 2 journals presented the lowest compliance with publishing transparency criteria, both with 9. The analysis concludes by warning about the need for publishing transparency to be a mandatory compliance standard for indexing or permanence in publication systems such as CONACYT.

Keywords: Publishing Transparency; Ethics of Scientific Communication; Education Journals;

Scientific Publications; Mexico 


\section{INTRODUCCIÓN}

- 1 objeto central de estudio de la presente investigación aborda la concepCión de la transparencia editorial en revistas científicas en el área de conocimiento de la Educación. Con carácter general, la transparencia editorial en revistas científicas viene asociada a la denominada ética de la comunicación científica como principio o pauta de la conducta vinculada al ámbito moral de las investigaciones, por cuanto los aspectos éticos y de transparencia son transversales a todo el proceso de investigación científica y editorial y, a su vez, forman parte de la credibilidad del canal de difusión.

Indudablemente, la calidad y la visibilidad de los contenidos de una revista están, de manera muy estrecha, vinculadas al proceso editorial, por ello las políticas editoriales de las publicaciones científicas preocupan a los editores. Son políticas que determinan los criterios de aceptación de manuscritos, las condiciones técnicas, los estándares normativos, el modelo de revisión por expertos (peer review), la periodicidad, la apertura de acceso a la publicación y también a los datos de investigación, etc. Esta información es relevante para decidir cómo deben publicar los investigadores en comunicación científica.

Es muy significativa la literatura publicada en los últimos años sobre investigaciones en torno a la responsabilidad ética en la gestión editorial. Mostramos algunos trabajos, a saber:

1. Respecto de los conflictos de intereses entre editores, autores y revisores de publicaciones científicas, las profesoras Tur-Viñes, Fonseca-Mora y Gutiérrez-San-Miguel (2012) analizan y revisan las recomendaciones éticas de las principales iniciativas internacionales, los organismos promotores y las recomendaciones específicas que se realizan para cada agente del proceso. Destacan cómo el grado de sensibilización frente a la ética de las publicaciones en cada ciencia y país depende de la tradición, el asociacionismo, la reputación y el grado de internacionalización de las revistas que configuran cada campo científico.

2. Un trabajo posterior de las citadas autoras propone conocer la perspectiva de los editores de revistas de Psicología, Educación y Comunicación en el contexto español. Es un estudio exploratorio que describe cuatro variables: facilidad de identificación de una mala praxis, frecuencia, gravedad, y opinión de los editores para un futuro próximo. Entienden por percepción editora el conjunto de experiencias, conocimientos, opiniones y creencias sobre aspectos éticos derivados de la gestión editorial (Fonseca-Mora, Tur-Viñes y Gutiérrez-San Miguel, 2014). 
3. Ahondando en el objeto de estudio del artículo anterior para las revistas científicas españolas de Comunicación, Educación y Psicología, las autoras configuran un escenario donde la necesidad de una práctica ética rigurosa adquiere cada vez más importancia al gestionar el proceso editorial. Los resultados de la investigación revelan los aspectos susceptibles de atención y mejora en el incipiente cambio de paradigma que se vislumbra para los editores al tener un tratamiento desigual, no consensuado y carente de estandarización (Gutiérrez San Miguel, Tur-Viñes y Fonseca-Mora, 2016).

4. Por último, Hernández-Ruiz (2016) analiza la política editorial antifraude de las revistas españolas y latinoamericanas indexadas en el JCR en Ciencias Sociales (2014). La inclusión de normas éticas en la política editorial científica lo concibe como una medida preventiva y disuasoria de conductas inapropiadas.

En efecto, existen organismos internacionales y comités como el Committee on Publication Ethics (COPE), el Council of Science Editors, el International Committee of Medical Journal Editors (ICJME), entre otros, que ofrecen un código ético y recomendaciones sobre cómo actuar en caso de detección de un fraude.

El código ético de una revista científica contiene la normativa pública y explícita que regula la transparencia y funcionamiento de los tres tipos de agentes participantes en las publicaciones académicas: los editores, los autores y los revisores. Puede destacarse, por ser de aplicación a todas las áreas de conocimiento, el Principles of Transparency and Best Practice in Scholarly Publishing (COPE, 2018), que incluye recomendaciones orientadas a los actores participantes en la publicación científica las cuales inciden sobre todo en los comités editoriales, que integran expertos idóneos en las distintas áreas de conocimiento de cada revista y en el proceso de revisión por pares, fundamental insumo en la calidad de los artículos de investigación que integran las publicaciones científicas.

Investigaciones recientes sobre comunicación académica insisten en la influencia que ejercen los editores, una influencia significativa en la misión de una revista y su contenido. El artículo titulado "Does the institutional diversity of editorial boards increase journal quality? The case economics field", de la prestigiosa revista Scientometrics, describe un caso empírico en el campo de la economía para explorar cómo la composición de los miembros del consejo editorial, en particular la diversidad en términos de institución, está relacionada con la calidad de la revista. 
El trabajo amplía el escaso conocimiento sobre la composición de equipos editoriales y su relevancia para la calidad de las revistas mediante el análisis de la correlación entre el índice de diversidad institucional y tres índices diferentes de calidad de las revistas. La implicación de este estudio se centra en la necesidad de un mayor esfuerzo para aumentar la diversidad en la composición de los equipos editoriales con el fin de garantizar la transparencia y promover la equidad (Dengsheng et al., 2020).

Finalmente, para Fatone et al. (2020) el desafío al que se enfrentan los editores y los miembros del consejo editorial consiste en promover transparencia de la investigación de acuerdo con los estándares de publicación, manteniendo el anonimato de autores y facilitando un proceso objetivo y justo de revisión por pares.

Basándonos en lo expuesto anteriormente, entendemos que la responsabilidad de transparencia editorial no sólo debe enfocarse desde la perspectiva de la ética sino más bien dirigirse hacia un proceso de gestión integral en cuanto a transparencia editorial en las revistas científicas con independencia de la o las disciplinas en que se ubiquen. Para conocer con detalle cómo las publicaciones ejecutan la transparencia editorial cotidianamente, a continuación presentamos los resultados de un estudio sobre este caso en revistas mexicanas de Educación con reconocida calidad científica.

\section{Metodología}

El análisis documental, definido como el análisis de información registrada en materiales duraderos escritos y visuales sujetos a sistematización, descripción e interpretación (Vázquez Navarrete, 2006: 74), es la metodología cualitativa utilizada en esta investigación.

El proceso metodológico consta de dos fases: en la primera se revisaron las fuentes documentales de alcance internacional Principles of Transparency and Best Practice in Scholarly Publishing (COPE, 2018) y Directrices para autores y traductores de artículos científicos publicados en inglés (EASE, 2018). Esta consulta derivó en la construcción de un listado con 15 criterios a evaluar para cualificar la transparencia editorial en las revistas científicas objeto de estudio de la investigación:

1. Sitio web con declaración de objetivos y alcance, lectores definidos, tipos de manuscritos que publica y números de identificación ISSN

2. Claridad en el nombre de la revista.

3. Declaración de proceso de revisión por pares, método y tiempos que no han de ser demasiado breves-. 
4. Claridad en la gestión de la revista (nombres de la entidad editora con dirección y el editor con filiación institucional).

5 Contar con órganos rectores como consejo editorial y/o comité científico externo (deberán incluirse los nombres completos y filiaciones institucionales de sus miembros).

6. Proporcionar información de contacto con la entidad que publica la revista.

7. Inclusión de la política de derechos de autor en las directrices para los autores y empleo de las licencias de los contenidos tanto en el sitio web de la publicación como en las versiones PDF y HTML de los artículos.

8. Declaratoria de cargos por publicación de artículos o en su caso de su ausencia.

9. Inclusión de pautas o directrices éticas que regulen la mala conducta de los autores en relación al plagio, la manipulación de resultados, los conflictos de interés, y la falsificación/fabricación de datos, entre otros aspectos.

10. Declaratoria de periodicidad con la que se publica la revista.

11. Modalidad de acceso a los contenidos de la revista -número íntegro o fraccionado- (suscripción, acceso abierto, etc.)

12. Señalamiento de archivado web y preservación digital de la publicación vía LOCKSS o sistema similar.

13. Especificación de fuentes de ingresos, subvenciones o patrocinios para la publicación de la revista.

14. Manifiesto de inclusión de publicidad acorde al contenido de la publicación en los casos en los que aplique.

15. Los artículos de investigación que sean publicados por la revista deberán contener los siguientes elementos:

a) Título

b) Lista de autores

c) Resumen

d) Palabras clave

e) Introducción

f) Métodos

g) Resultados

h) Discusión

i) Agradecimientos -en los casos en que aplique-

j) Referencias 
Los criterios 1 al 14 son imprescindibles en cuanto a la forma de la publicación y se eligieron con base en los Principles of Transparency and Best Practice in Scholarly Publishing (COPE, 2018), mientras que el criterio 15 es un indicador necesario en cuanto a fondo, mismo que aparece descrito en las $D i$ rectrices para autores y traductores de artículos cientificos publicados en inglés (EASE, 2018).

En la segunda fase del análisis documental, se aplicaron los criterios anteriormente mencionados a una población de 18 revistas científicas mexicanas de Educación indizadas en el Sistema de Clasificación de Revistas Mexicanas de Ciencia y Tecnología del Consejo Nacional de Ciencia y Tecnología (Conacyt). Este sistema se autodefine en el Manual del Sistema de Clasificación de Revistas Mexicanas de Ciencia y Tecnología como un "instrumento de política pública que, mediante el registro selectivo y la evaluación periódica de las revistas científicas editadas en formato electrónico en México, busca elevar su calidad, visibilidad e impacto y, de este modo, fomentar la difusión y la divulgación de la ciencia y la tecnología generada en el país” (Conacyt, 2019). La evaluación de estas revistas es efectuada mediante pares evaluadores internacionales, especialistas en edición científica, usando el método de ciego simple y tomando en cuenta 26 criterios organizados en seis dimensiones: política y gestión editorial, calidad de contenido, nivel de citación, cumplimiento de la frecuencia de publicación, accesibilidad, y visibilidad internacional.

El grupo de revistas estudiado comprende publicaciones que abordan temáticas ad hoc a dicho dominio de conocimiento, a saber: Educación especializada por disciplinas, Subdisciplinas de la Educación, Evaluación educativa, Ciencias de la Educación, Filosofía de la Educación, Pedagogía, Psicología educativa y Tecnología educativa. Los resultados de esta última etapa se exponen a continuación.

\section{PresentaCión y ANÁlisis de RESUltados}

La Tabla 1 presenta el número total de criterios de transparencia editorial que han sido cumplidos, parcialmente cumplidos e incumplidos por las revistas mexicanas de Educación indizadas en el Sistema de Clasificación de Revistas Mexicanas de Ciencia y Tecnología con fecha de corte 24 de julio de 2020. Con el objetivo de proporcionar mayor información sobre estas publicaciones se incluyen datos complementarios, como nombre de la institución u organización editora, su ubicación actual en la web, periodicidad e incorporación en otros sistemas de indización que evidencian la calidad de la revista. 


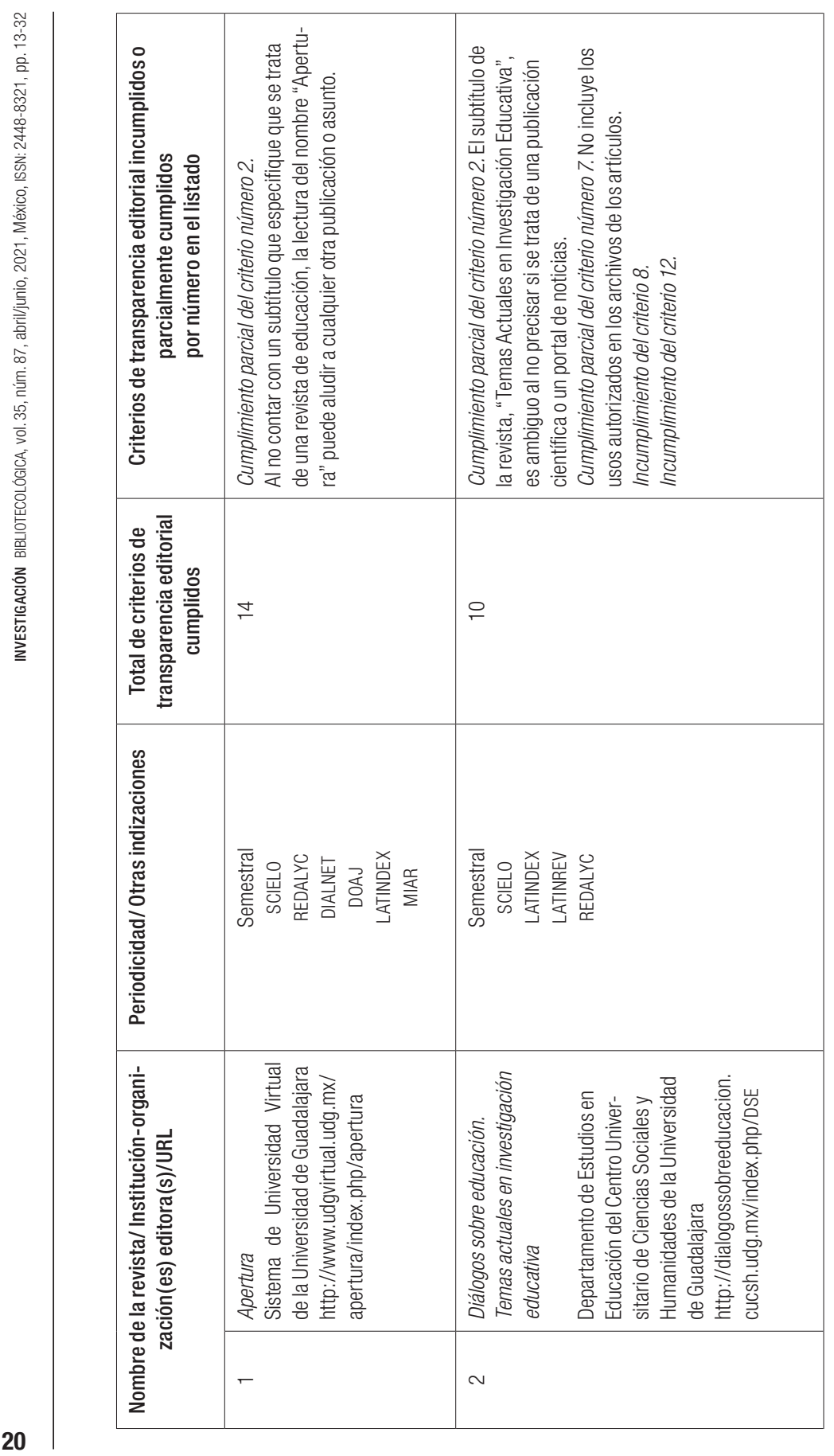




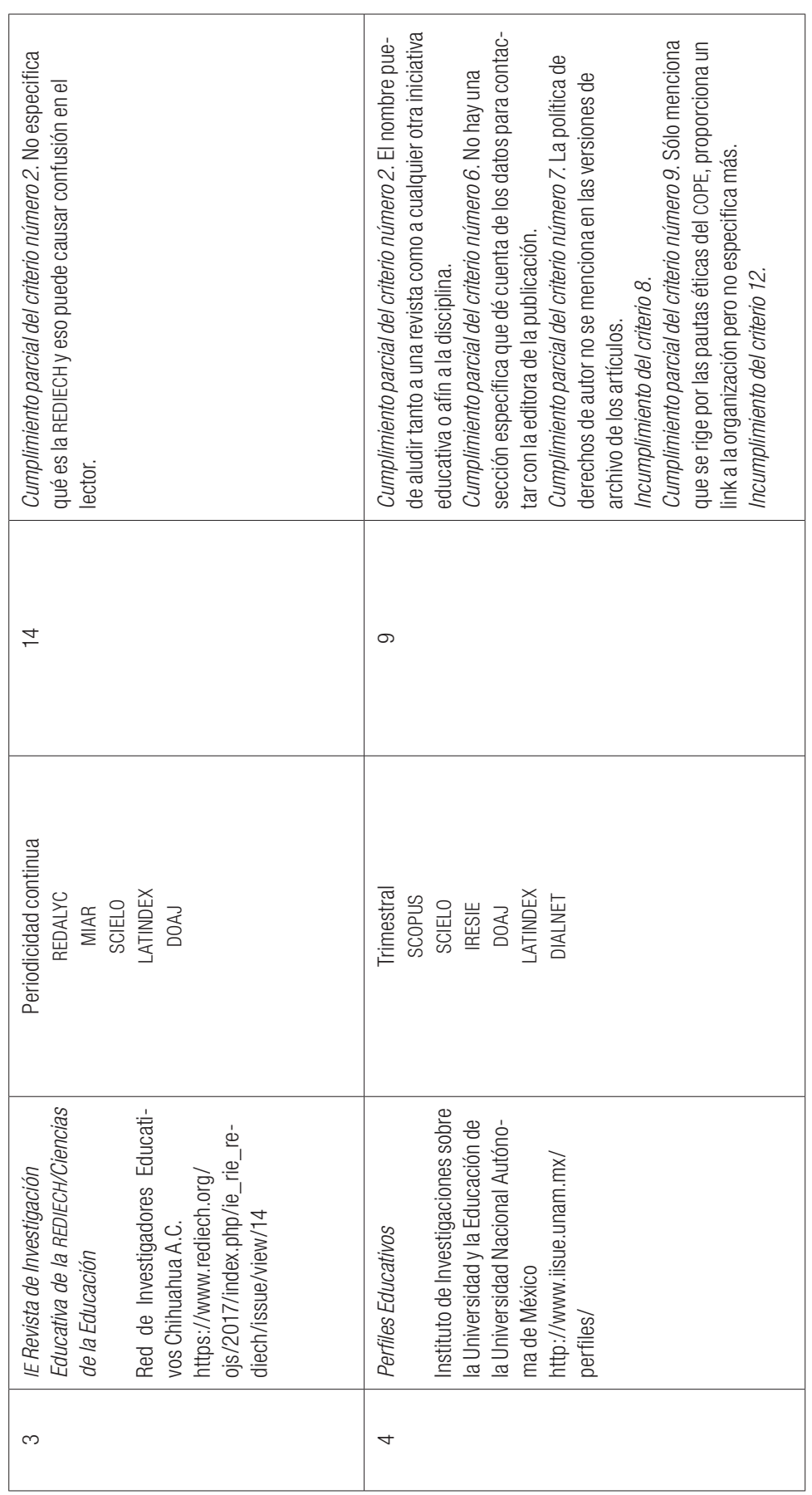




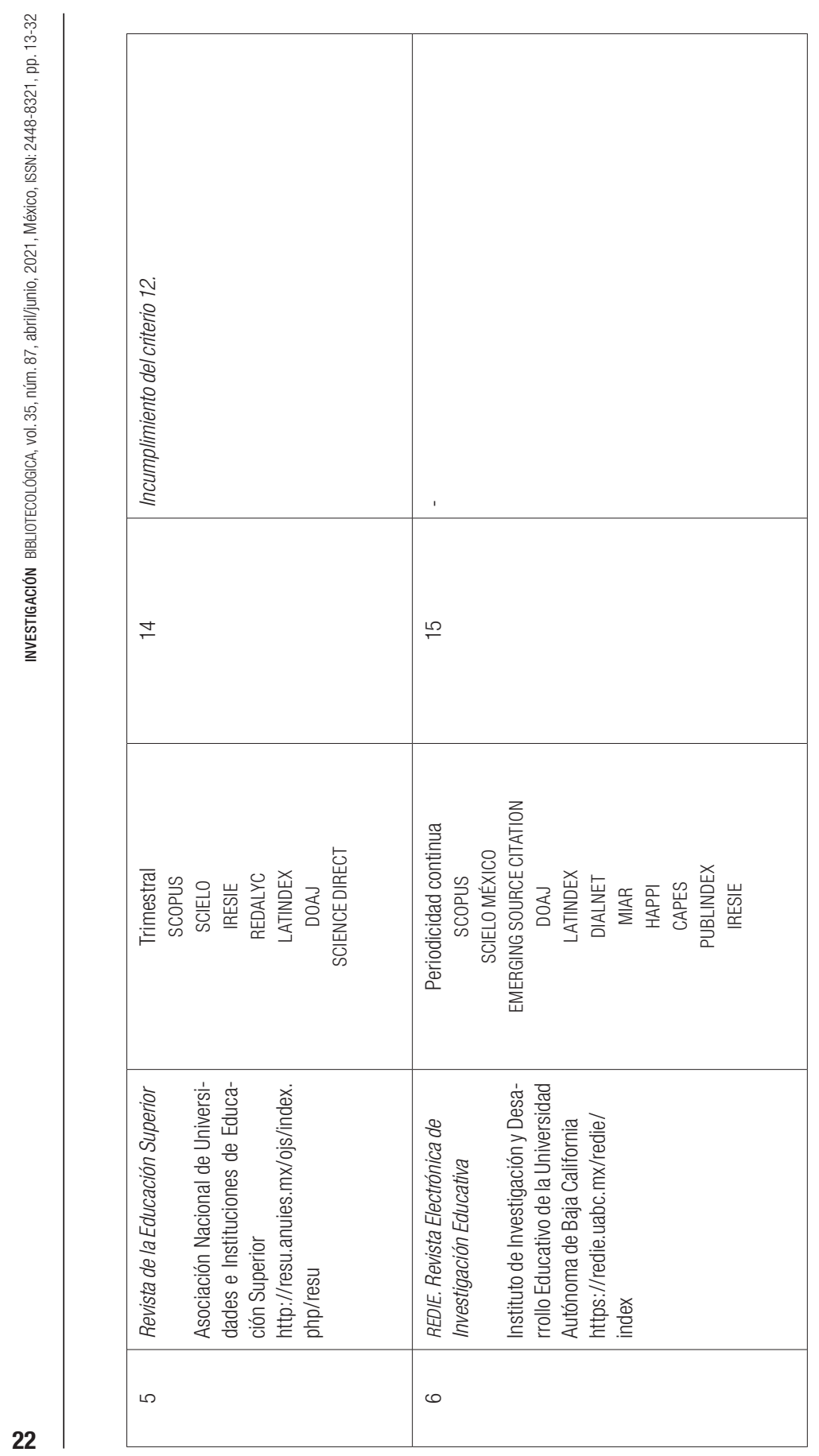




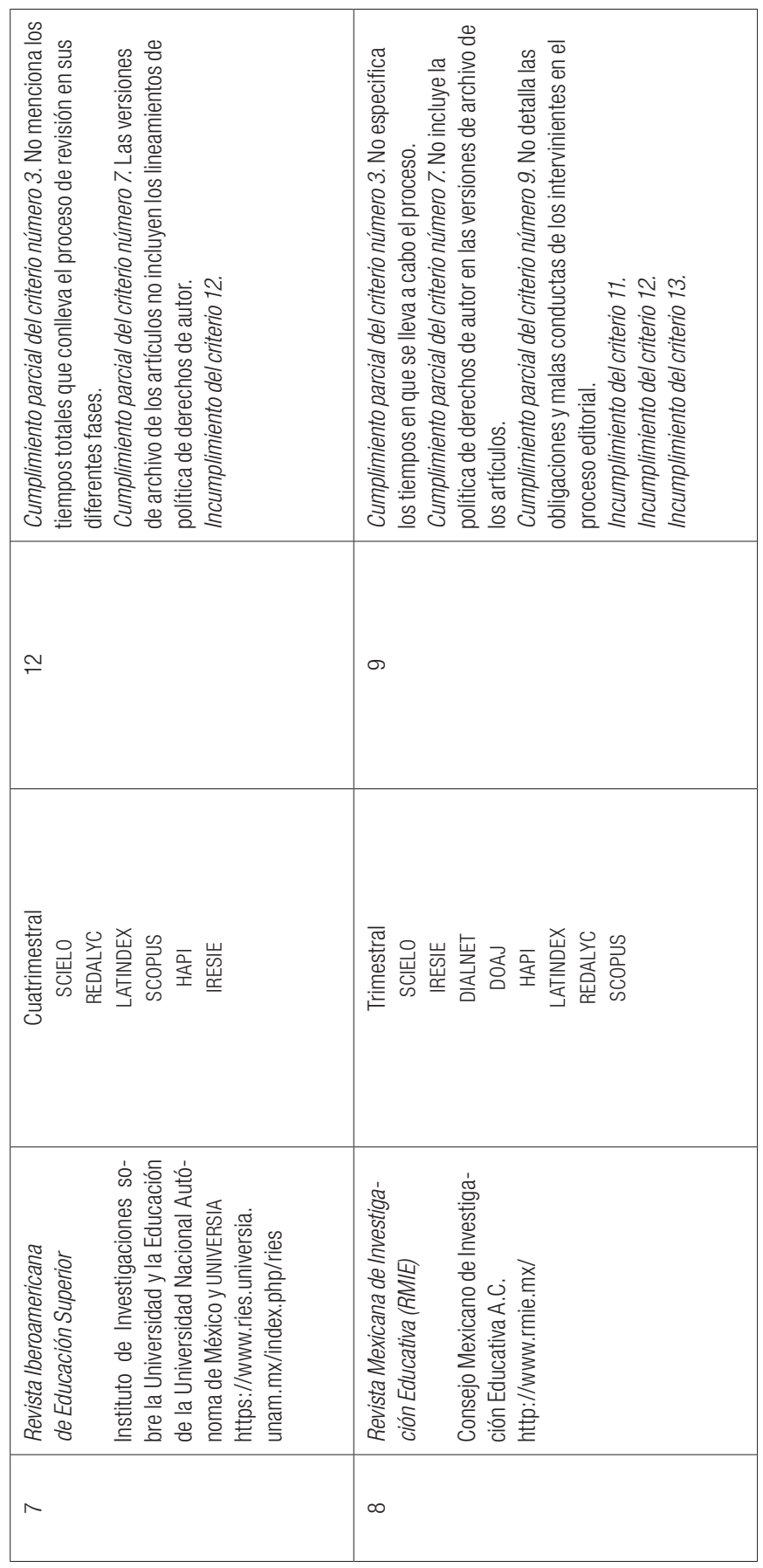




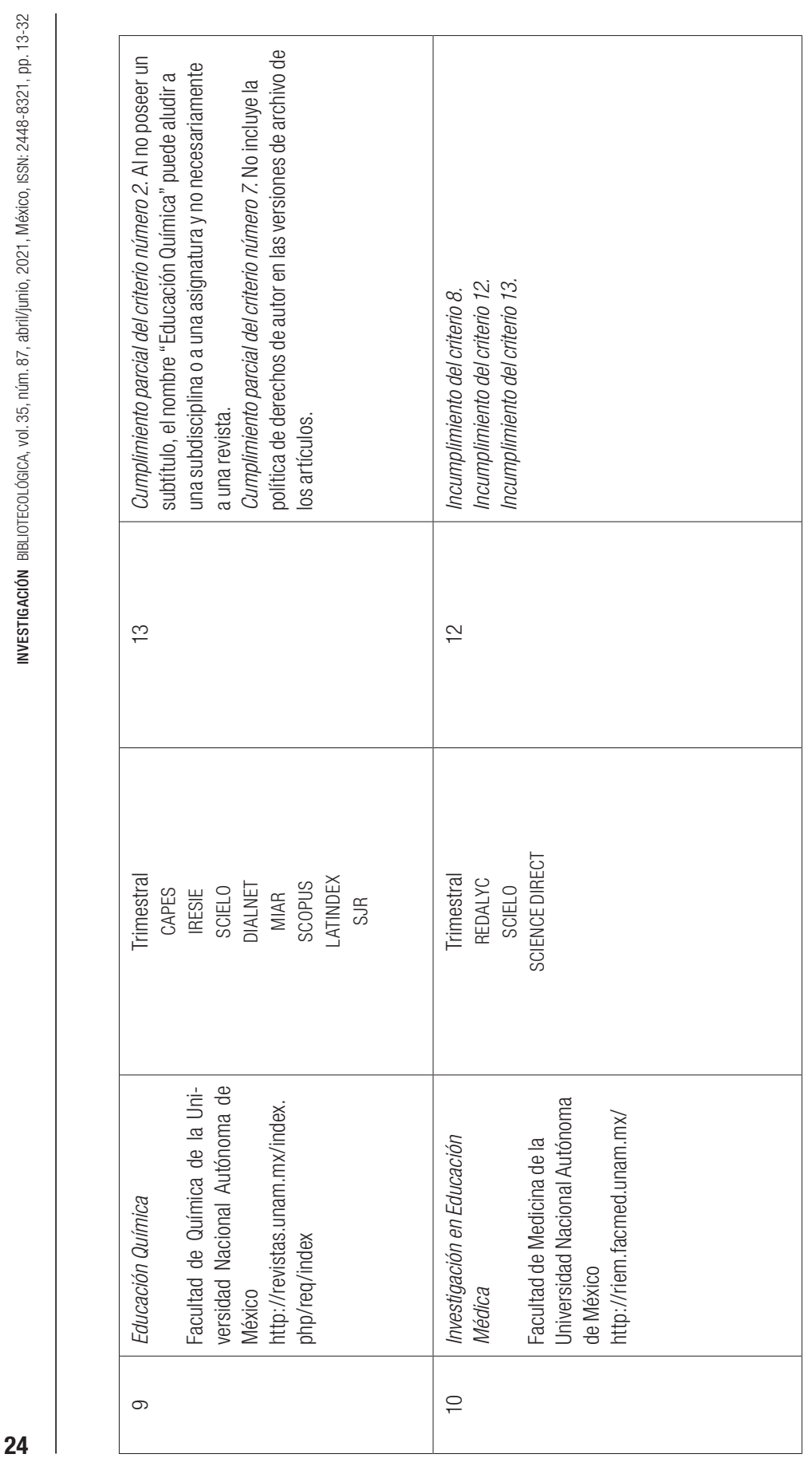




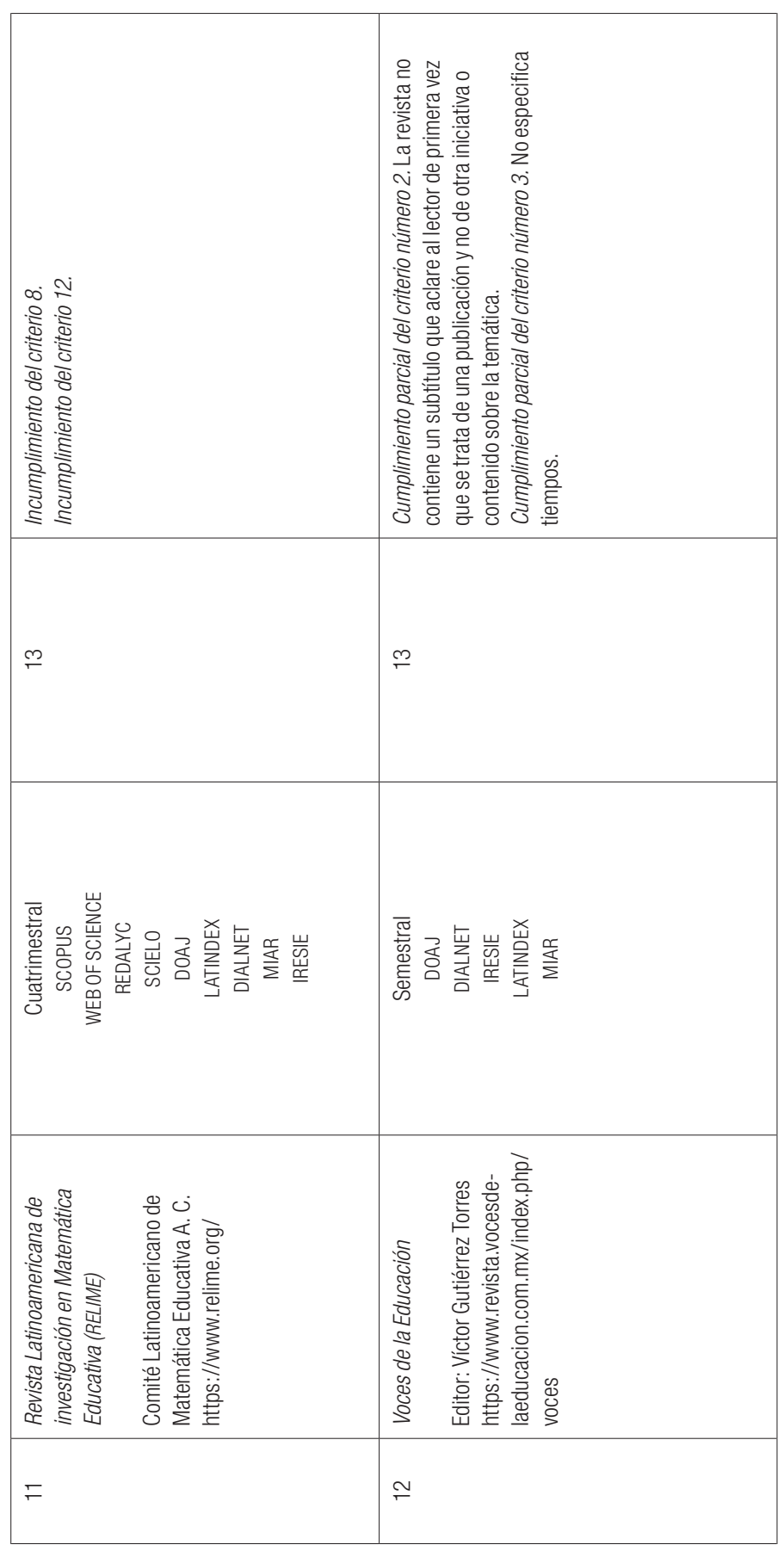




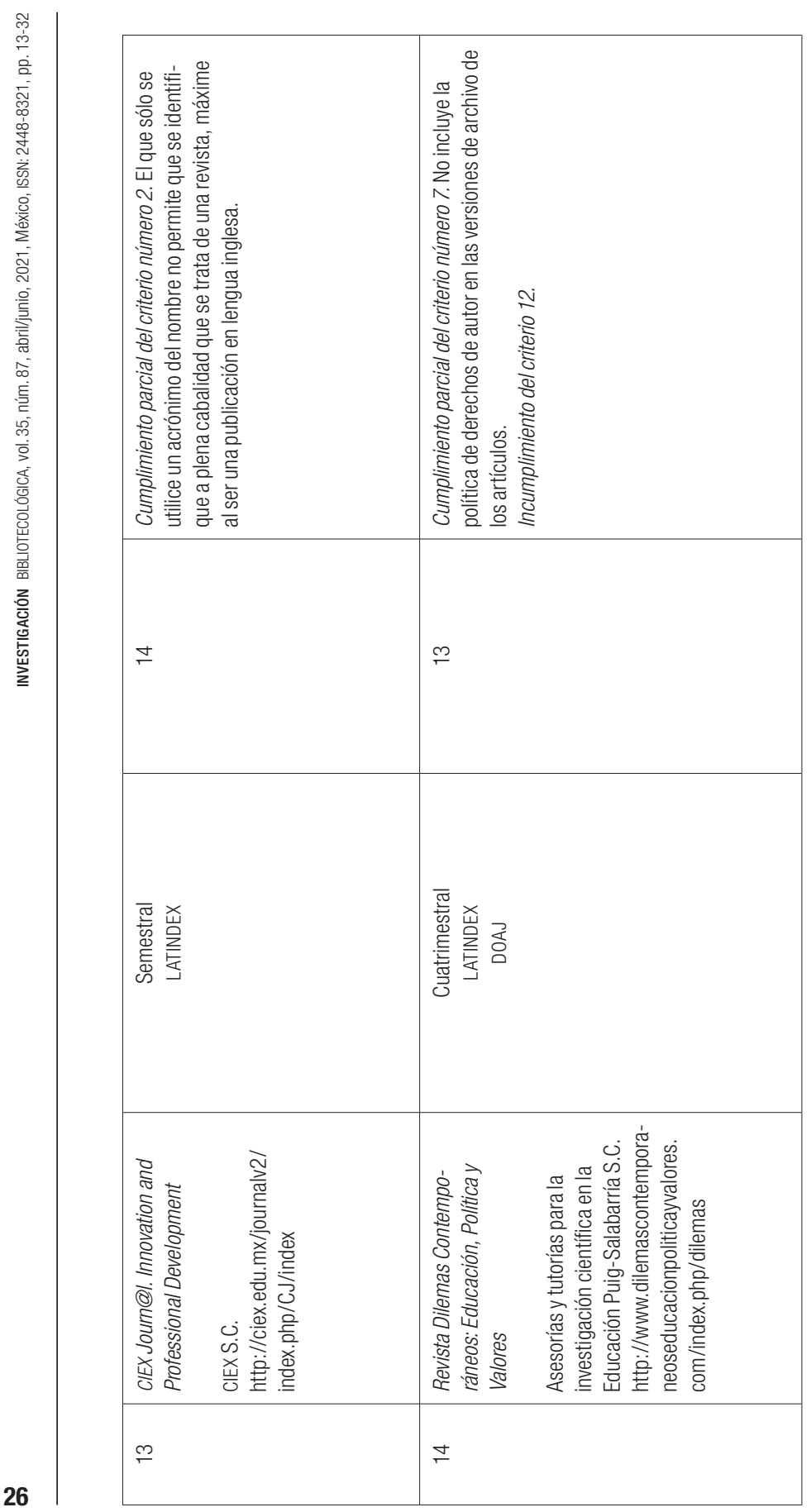




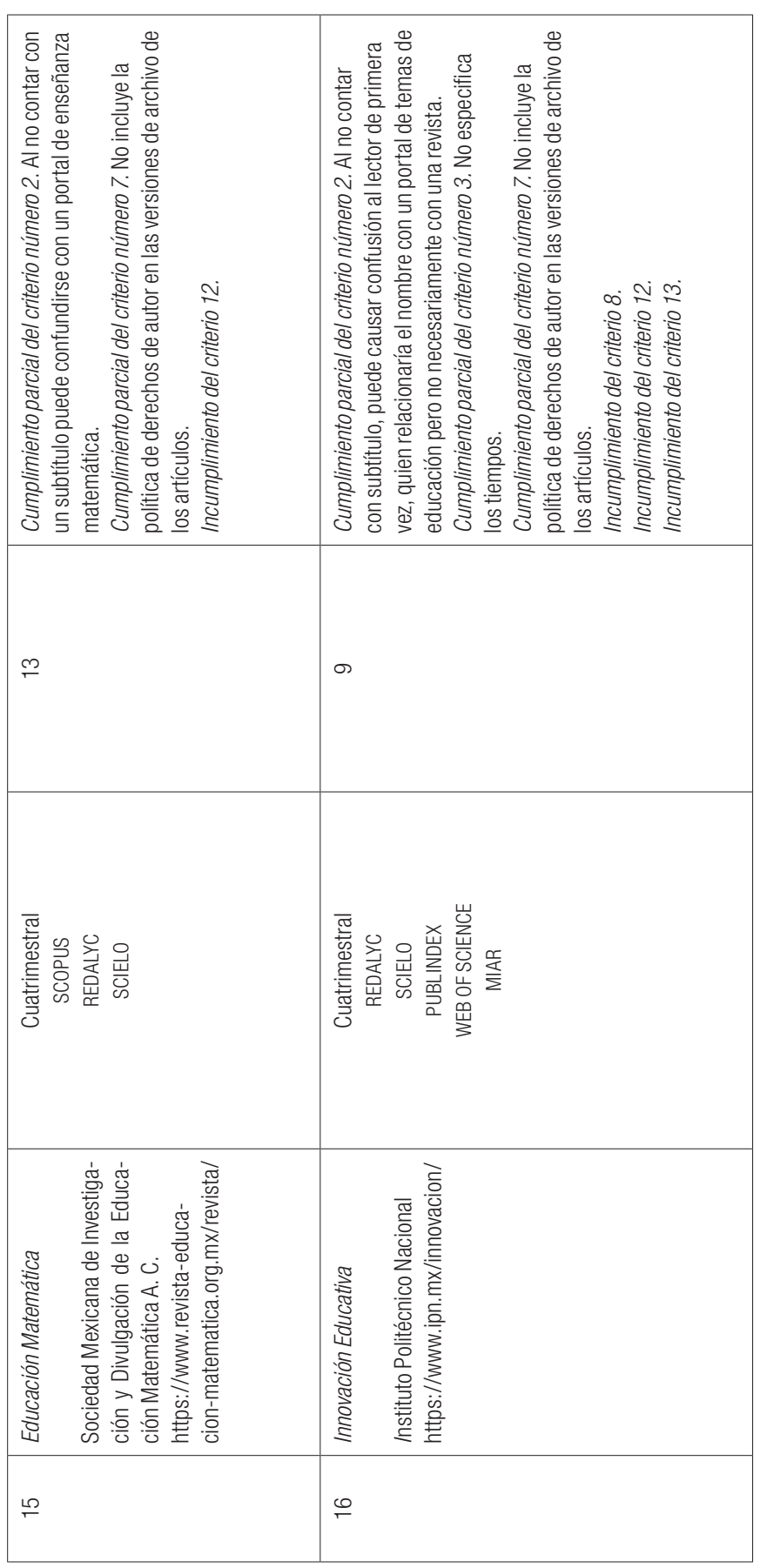




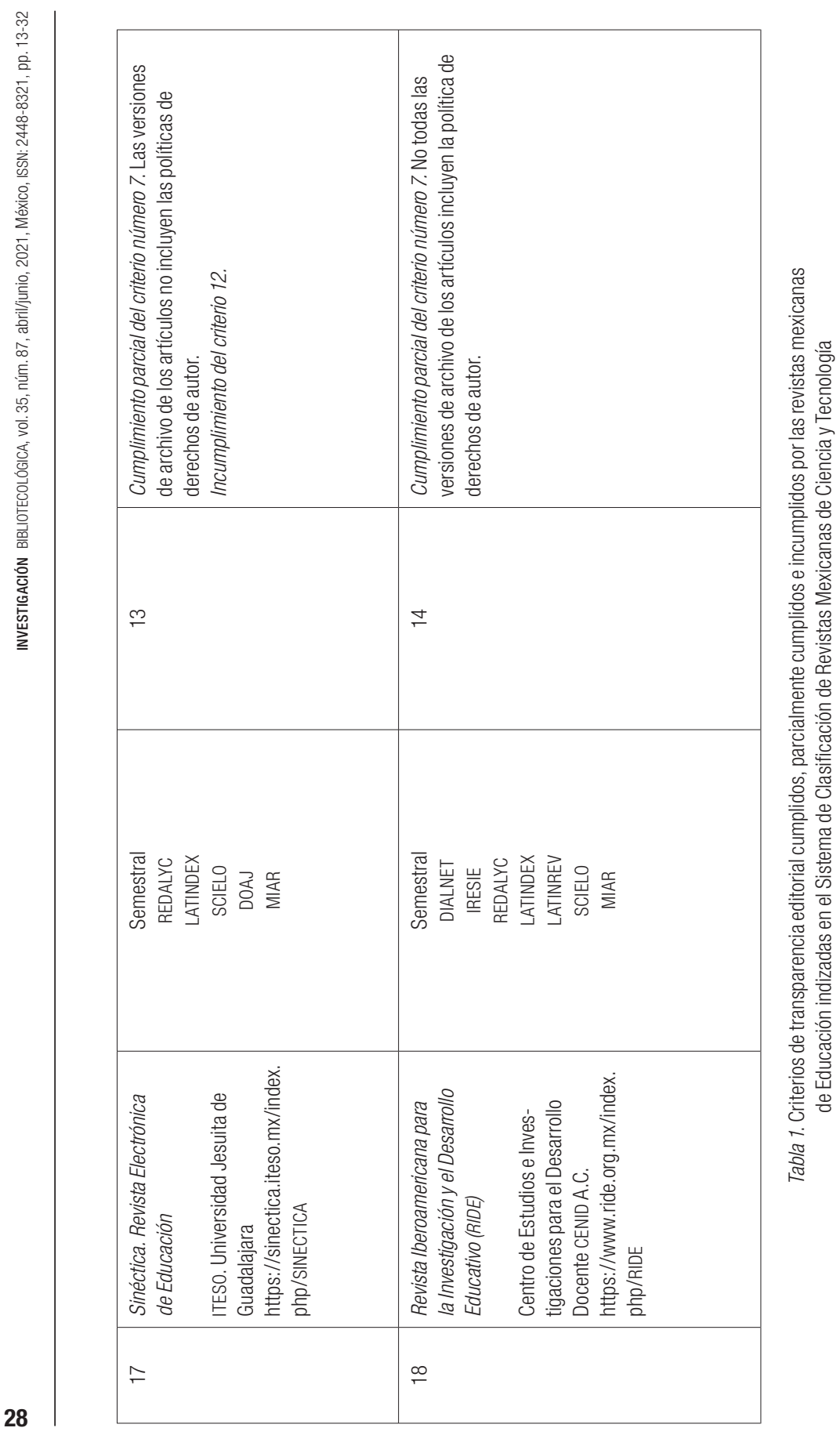


De las 18 revistas mexicanas de Educación analizadas, se encontró que sólo una, la REDIE. Revista Electrónica de Investigación Educativa, cumple con los 15 criterios de transparencia editorial establecidos. En este caso estaríamos tratando de una publicación con una gestión integral en cuanto a transparencia editorial, situación que puede explicarse por lo siguiente: la REDIE ha sido una revista pionera en la incorporación de formatos de distribución de sus contenidos y de la inclusión de nuevas prácticas editoriales en el país, como la periodicidad continua. Empero, es de destacar que cinco revistas Revista Iberoamericana para la Investigación y el Desarrollo Educativo (RIDE), Revista de la Educación Superior, CIEX Journ@l. Innovation and Professional Development, IE Revista de Investigación Educativa de la REDIECH/Ciencias de la Educación y Apertura-, 28 \% del total estudiado, presentan una alta transparencia editorial al cumplir con 14 de los 15 criterios recomendados. Estas publicaciones presentaron deficiencias en lo que respecta a la inclusión de políticas de derechos de autor en todas las versiones de sus artículos (Revista Iberoamericana para la Investigación y el Desarrollo Educativo); señalamiento de archivado web y preservación digital de la publicación vía LOCKSS o sistema similar (Revista de la Educación Superior) y claridad en el nombre de la revista, criterio en el que cumplieron sólo parcialmente tres de las cinco publicaciones (CIEX Journ@l. Innovation and Professional Development, IE Revista de Investigación Educativa de la REDIECH/Ciencias de la Educación y Apertura). Las revistas Innovación Educativa y Revista Mexicana de Investigación Educativa (RMIE) son las publicaciones con menor cumplimiento de criterios de transparencia editorial, ambas con nueve de 15 criterios cumplidos. Esto llama la atención ya que la primera se encuentra indizada en la Web of Science y la segunda en Scopus, tal situación puede plantear la hipótesis de que los sistemas de evaluación de revistas más robustos adolecen de falta de transparencia editorial como criterio de ponderación decisivo para su incorporación.

Adicionalmente, es preocupante que los criterios en que las revistas mexicanas de Educación estudiadas presentan mayor incumplimiento o cumplimiento parcial sean el 12, señalamiento de archivado web y preservación digital de la publicación vía LOCKSS o sistema similar (11 publicaciones), y el 7, la inclusión de la política de derechos de autor en las directrices para los autores así como el empleo de las licencias de los contenidos tanto en el sitio web de la publicación como en las versiones PDF y HTML de los artículos (10 revistas).

Estos datos exponen la vulnerabilidad tan alta en la que se encuentran los contenidos de aquellas publicaciones para su conservación y reutilización futuras, así como la imprudencia o desconocimiento de los responsables edi- 
toriales sobre la necesidad-obligatoriedad de incorporar las licencias de uso en cada una de las versiones de sus contribuciones, ya que es común que los lectores lleguen al contenido concreto sin consultar la revista íntegra. Efectuar esta práctica contribuye al respeto por los derechos de autor y a la valoración de los términos y condiciones con la que ha de emplearse éticamente la información que se difunde en internet.

\section{Discusión}

Los resultados anteriores han permitido comprobar la adopción de criterios de transparencia editorial de manera amplia por las revistas de Educación mexicanas indizadas en el Sistema de Clasificación de Revistas del Conacyt. Con ello podemos afirmar que se dirigen de manera efectiva hacia una gestión editorial integral, empero, aún hace falta trabajo por parte de sus equipos editoriales. Si bien las referidas publicaciones han adoptado de manera casi unilateral las directrices del COPE, éstas siguen percibiéndose como aspectos éticos y no de política y gestión editorial de la revista, como en realidad lo son. Además, destacamos y alertamos sobre el hecho de que los lineamientos de este organismo internacional de editores no contemplen el contenido como objeto/sujeto de transparencia cuando va directamente ligado a los temas de ética: autoría, contribución, conflictos de interés y fuentes de financiamiento. Ante esta carencia, las autoras del presente trabajo optamos por incluirlo como el criterio 15 , previa consulta de textos y otros materiales sobre ética de la comunicación científica. De igual modo, creemos que el esquema propuesto por COPE debe ser revisado y actualizado constantemente, ya que las revistas científicas se encuentran en una constante evolución; por poner un ejemplo, la obligatoriedad de que los artículos u otros documentos contengan DOI no se menciona pese a que se vincula con el criterio 12 , realtivo al señalamiento de archivado web y preservación digital de la publicación vía LOCKSS o sistema similar.

\section{Conclusiones}

En la revisión de las fuentes documentales sobre la transparencia editorial en revistas científicas observamos que es un tema de investigación tratado en foros y publicaciones asociados a la ética de la comunicación académica. Sin embargo, es muy aconsejable integrar la transparencia editorial como parte de la gestión editorial de las revistas científicas no sólo por lo expuesto en 
esta investigación sino por su aportación al ecosistema de la comunicación académica en su conjunto. Aunque sí existen directrices generales a nivel internacional, no tienen carácter formal en los índices o sistemas de evaluación de revistas científicas.

Estudiar las revistas mexicanas de Educación del Sistema de Clasificación de Revistas del Conacyt ha permitido efectuar un primer acercamiento sobre la transparencia editorial de dichas publicaciones científicas contribuyendo a su mejora. Es un precedente de investigación sobre la materia a nivel nacional extensivo al contexto latinoamericano. Por último, manifestar la necesidad del trabajo colaborativo entre editores para definir los instrumentos de transparencia editorial.

\section{REFERENCIAS}

Conacyt (Consejo Nacional de Ciencia y Tecnología). 2019. Manual del Sistema de Clasificación de Revistas Mexicanas de Ciencia y Tecnología. Ciudad de México: Conacyt. http://www.revistascytconacyt.mx/manual-sistema-crmcyt2019.pdf

COPE (Committee on Publication Ethics). 2018. Principles of Transparency and Best Practice in Scholarly Publishing. Hampshire: COPE.

https://publicationethics.org/resources/guidelines-new/principles-transparency-and-best-practice-scholarly-publishing

Dengsheng Wu, Xiaoli Lu, Jianping Li y Jing Li. 2020. "Does the institutional diversity of editorial boards increase journal quality? The case economics field". Scientometrics 124: 1579-1597.

https://doi.org/10.1007/s11192-020-03505-6

EASE (European Association of Science Editors). 2018. Directrices para autores y traductores de artículos cientificos publicados en inglés. Londres: EASE. https://doi.org/10.20316/ESE.2018.44.e1.sp

Fatone, Stefania, Michael P. Dillon, Brian J. Hafner y Nerrolyn Ramstrand. 2020. "The challenges of double-blind peer review in an era of increasing research transparency". Prosthetics and Orthotics International 44 (4): 189-191. https://doi.org/10.1177\%2F0309364620937864

Fonseca-Mora, María Carmen, Victoria Tur-Viñes y Begoña Gutiérrez-San Miguel. 2014. "Ética y revistas científicas españolas de Comunicación, Educación y Psicología: la percepción editora”. Revista Española de Documentación Científica 37 (4). http://dx.doi.org/10.3989/redc.2014.4.1151

Gutiérrez San Miguel, Begoña; Victoria Tur-Viñes y María Carmen Fonseca-Mora. 2016. "Principios éticos de los editores en las revistas científicas españolas de Comunicación, Educación y Psicología”. Ibersid 1 (2): 53-60. https://www.ibersid.eu/ojs/index.php/ibersid/article/view/4310

Hernández-Ruiz, Alejandra. 2016. "Antifraud Editorial Policy in Spanish and Latin American Scientific Publication: JCR Social Sciences Edition”. Comunicar 48: 19-27. https://doi.org/10.3916/C48-2016-02 
Tur-Viñes, Victoria, María Carmen Fonseca-Mora y Begoña Gutiérrez-San-Miguel. 2012. "Ética de la publicación científica: iniciativas y recomendaciones". El Profesional de la Información 21 (5): 491-497. http://eprints.rclis.org/17726/

Vázquez Navarrete, María Luisa (coord.). 2006. Introducción a las técnicas cualitativas de investigación aplicadas en salud. Barcelona: Universidad Autónoma de Barcelona.

Para citar este texto:

Fernández Bajón, María Teresa y Jenny Teresita Guerra González. 2021. "Transparencia editorial en revistas científicas mexicanas de educación: hacia una gestión integral de las políticas editoriales en las publicaciones periódicas científicas". Investigación Bibliotecológica: archivonomía, bibliotecología e información 35 (87): 13-32.

http://dx.doi.org/10.22201/iibi.24488321xe.2021.87.58340 